Bull. Austral. Math. Soc.

Vol. 64 (2001) [469-474]

\title{
ON RECURRENCE RELATIONS FOR BERNOULLI AND EULER NUMBERS
}

\author{
Ching-Hua Chang and Chung-Wei Ha
}

We obtain a class of recurrence relations for the Bernoulli numbers that includes a recurrence formula proved recently by $M$. Kaneko. Analogous formulas are also derived for the Euler and Genocchi numbers.

\section{INTRODUCTION}

The Bernoulli polynomials $B_{n}(x)$ and the Euler polynomials $E_{n}(x)(n=0,1,2, \ldots)$ may be computed successively by means of the formulas

$$
\sum_{k=0}^{n}\left(\begin{array}{c}
n+1 \\
k
\end{array}\right) B_{k}(x)=(n+1) x^{n}, \quad E_{n}(x)+\sum_{k=0}^{n}\left(\begin{array}{l}
n \\
k
\end{array}\right) E_{k}(x)=2 x^{n} .
$$

Thus the corresponding Bernoulli and Euler numbers, defined respectively by $B_{n}=$ $B_{n}(0)$ and $E_{n}=2^{n} E_{n}(1 / 2)(n=0,1,2, \ldots)$, satisfy $B_{0}=E_{0}=1$ and the recurrence relations

$$
\sum_{k=0}^{n}\left(\begin{array}{c}
n+1 \\
k
\end{array}\right) B_{k}=0, \quad E_{n}+2^{n-1} \sum_{k=0}^{n-1}\left(\begin{array}{l}
n \\
k
\end{array}\right) \frac{E_{k}}{2^{k}}=1 \quad(n \geqslant 1) .
$$

Two important properties of Bernoulli and Euler polynomials we shall make use of below are

$$
\begin{array}{ll}
B_{n+1}^{\prime}(x)=(n+1) B_{n}(x), & E_{n+1}^{\prime}(x)=(n+1) E_{n}(x) \\
B_{n}(x+1)-B_{n}(x)=n x^{n-1}, & E_{n}(x+1)+E_{n}(x)=2 x^{n} .
\end{array}
$$

We refer to [1] for a good account of the properties of $B_{n}(x), E_{n}(x)$ and the corresponding Bernoulli and Euler numbers.

Recently a new recurrence formula for Bernoulli numbers was obtained in Kaneko [6], for which two proofs were given (see also Satoh [8]). In this note we offer a proof of Kaneko's formula which is simpler than those given in $[6,8]$ and, significantly, leads to a general class of recurrence relations for Bernoulli numbers. Analogous formulas for Euler and Genocchi numbers are also derived. Other interesting recurrence relations for Bernoulli numbers may be found in $[3,5]$ and $[7, p .122]$.

Received 9th April, 2001

Copyright Clearance Centre, Inc. Serial-fee code: 0004-9727/01 \$A2.00+0.00. 


\section{Two Lemmas}

We first give two simple properties involving Bernoulli and Euler polynomials on which our results are based.

LEMMA 1 . For an integer $n \geqslant 0$, the polynomials $P_{n}(x)$ and $Q_{n}(x)$ of degree $2 n$ defined by

$$
P_{n}(x)=\sum_{j=0}^{n}\left(\begin{array}{l}
n \\
j
\end{array}\right) B_{n+j}(x) \quad \text { and } \quad Q_{n}(x)=\sum_{j=0}^{n}\left(\begin{array}{l}
n \\
j
\end{array}\right) E_{n+j}(x)
$$

are even functions.

Proof: It follows from (4) that

$$
\begin{aligned}
P_{n}(x+1)-P_{n}(x) & =\sum_{j=0}^{n}\left(\begin{array}{l}
n \\
j
\end{array}\right)\left\{B_{n+j}(x+1)-B_{n+j}(x)\right\} \\
& =\sum_{j=0}^{n}\left(\begin{array}{l}
n \\
j
\end{array}\right)(n+j) x^{n+j-1}=n x^{n-1}(x+1)^{n-1}(2 x+1)
\end{aligned}
$$

and

$$
\begin{aligned}
Q_{n}(x+1)+Q_{n}(x) & =\sum_{j=0}^{n}\left(\begin{array}{l}
n \\
j
\end{array}\right)\left\{E_{n+j}(x+1)+E_{n+j}(x)\right\} \\
& =\sum_{j=0}^{n}\left(\begin{array}{l}
n \\
j
\end{array}\right) 2 x^{n+j}=2 x^{n}(x+1)^{n} .
\end{aligned}
$$

For an integer $k \geqslant 0$ substituting $x=k$ and $x=-k-1$ into (5) and (6) we have

$$
\begin{aligned}
P_{n}(k+1)-P_{n}(k) & =P_{n}(-k-1)-P_{n}(-k), \\
Q_{n}(k+1)+Q_{n}(k) & =Q_{n}(-k-1)+Q_{n}(-k),
\end{aligned}
$$

and so by induction for all integers $k \geqslant 1$

$$
P_{n}(k)=P_{n}(-k), \quad Q_{n}(k)=Q_{n}(-k)
$$

The lemma now follows as both $P_{n}(x)$ and $Q_{n}(x)$ are polynomials.

LEMMA 2. For an integer $n \geqslant 0$

$$
\begin{aligned}
P_{n}(x)-P_{n}(1-x) & =\frac{d}{d x}\left[x^{n}(x-1)^{n}\right] \\
Q_{n}(x)+Q_{n}(1-x) & =2 x^{n}(x-1)^{n}
\end{aligned}
$$


Proof: Replacing $x$ by $-x$ in (5) it follows from Lemma 1 that

$$
P_{n}(x)=P_{n}(-x)=P_{n}(1-x)+\frac{d}{d x}\left[x^{n}(x-1)^{n}\right] .
$$

Using (6) instead we have similarly

$$
Q_{n}(x)=Q_{n}(-x)=-Q_{n}(1-x)+2 x^{n}(x-1)^{n} .
$$

We also need the following formula to evaluate certain derivatives. For integers $0 \leqslant m \leqslant n$

$$
\frac{1}{(2 m) !} \frac{d^{2 m}}{d x^{2 m}}\left[x^{n}(x-1)^{n}\right]_{x=1 / 2}=\left(-\frac{1}{4}\right)^{n-m}\left(\begin{array}{c}
n \\
m
\end{array}\right) .
$$

This follows from the Leibniz rule and the equality

$$
\sum_{k=0}^{2 m}(-1)^{k}\left(\begin{array}{l}
n \\
k
\end{array}\right)\left(\begin{array}{c}
n \\
2 m-k
\end{array}\right)=(-1)^{m}\left(\begin{array}{l}
n \\
m
\end{array}\right)
$$

(see [7, p.14]).

\section{RecurRence ReLations}

The main result of this note is the following. We denote for integers $m, n \geqslant 0$, $[n-m]_{+}=\max \{n-m, 0\}$.

THEOREM 1. Let $n \geqslant 1$ be an integer. Then for any integer $m \geqslant 0$

(a)

$$
\begin{aligned}
& \sum_{k=[n-m]_{+}}^{2 n}\left(\begin{array}{c}
m+n+1 \\
m-n+k
\end{array}\right)\left(\begin{array}{c}
2 m+k+1 \\
k
\end{array}\right) B_{k}=0 \\
& \sum_{k=[n-m]_{+}}^{2 n}\left(\begin{array}{c}
m+n+1 \\
m-n+k
\end{array}\right)\left(\begin{array}{c}
2 m+k+1 \\
k
\end{array}\right) \frac{B_{k}}{2^{k}}=(-1)^{n} \frac{m+1}{2^{2 n+1}}\left(\begin{array}{c}
m+n+1 \\
n
\end{array}\right) .
\end{aligned}
$$

Proof: Let $m \geqslant 0$ be given. Applying (3) repeatedly we obtain the $(2 m+1)^{\text {th }}$ derivative of $P_{m+n+1}(x)$, which by Lemma 1 vanishes at $x=0$. Dividing the resulting summation by $(2 m+1)$ ! we have

$$
\sum_{j=[m-n]_{+}}^{m+n}\left(\begin{array}{c}
m+n+1 \\
j
\end{array}\right)\left(\begin{array}{c}
m+n+j+1 \\
2 m+1
\end{array}\right) B_{n-m+j}=0
$$

as $B_{j}=0$ for odd $j \geqslant 3$. We have (a) by substituting $j=m-n+k$. 
In a similar way we calculate the $(2 m+1)^{\text {th }}$ derivative of the expression $P_{m+n+1}(x)$ $-P_{m+n+1}(1-x)$ and evaluate at $x=1 / 2$. Dividing the resulting summation by $(2 m+1)$ ! we obtain (b) by (7), (9) and the formula in (a) as $B_{j}(1 / 2)=\left(1 / 2^{j-1}\right) B_{j}-B_{j}$ for $j \geqslant 0$.

A feature in the formulas obtained in Theorem 1 as distinct from some known results is the appearance in the coefficients of an arbitrarily chosen integer $m \geqslant 0$, by which the number of terms in the recurrence may be adjusted. The same remark applies also to those obtained in Theorems 2 and 3 below. Particularly interesting are the special cases when $m=0$ and $m=n$. We state them separately in the following result.

COROLLARY 1. For an integer $n \geqslant 1$

$$
\begin{aligned}
\sum_{k=n}^{2 n}\left(\begin{array}{c}
n+1 \\
k-n
\end{array}\right)(k+1) B_{k} & =0 \\
\sum_{k=n}^{2 n}\left(\begin{array}{c}
n+1 \\
k-n
\end{array}\right)(k+1) \frac{B_{k}}{2^{k}} & =(-1)^{n} \frac{n+1}{2^{2 n+1}} ; \\
\sum_{k=0}^{2 n}\left(\begin{array}{c}
2 n+k+1 \\
2 k
\end{array}\right)\left(\begin{array}{c}
2 k \\
k
\end{array}\right) B_{k} & =0 ; \\
\sum_{k=0}^{2 n}\left(\begin{array}{c}
2 n+k+1 \\
2 k
\end{array}\right)\left(\begin{array}{c}
2 k \\
k
\end{array}\right) \frac{B_{k}}{2^{k}} & =(-1)^{n} \frac{n+1}{2^{2 n+1}}\left(\begin{array}{c}
2 n+1 \\
n
\end{array}\right) .
\end{aligned}
$$

In deriving (c) and (d), we use the equality

$$
\left(\begin{array}{c}
2 n+1 \\
k
\end{array}\right)\left(\begin{array}{c}
2 n+k+1 \\
k
\end{array}\right)=\left(\begin{array}{c}
2 n+k+1 \\
2 k
\end{array}\right)\left(\begin{array}{c}
2 k \\
k
\end{array}\right)
$$

Kaneko's formula is now recovered in Corollary 1(a).

THEOREM 2. Let $n \geqslant 1$ be an integer. Then for any integer $m \geqslant 0$

$$
\sum_{k=[n-m]_{+}}^{2 n}\left(\begin{array}{c}
m+n \\
m-n+k
\end{array}\right)\left(\begin{array}{c}
2 m+k \\
k
\end{array}\right) \frac{E_{k}}{2^{k}}=\left(-\frac{1}{4} \cdot\right)^{n}\left(\begin{array}{c}
m+n \\
n
\end{array}\right) .
$$

Proof: Let $m \geqslant 0$ be given. We calculate the $(2 m)^{\text {th }}$ derivative of the expression $Q_{m+n}(x)+Q_{m+n}(1-x)$ using (3) as in the proof of Theorem 1 and evaluate at $x=1 / 2$. Dividing the resulting summation by $(2 m)$ ! we have by (8) and (9)

$$
\sum_{j=[m-n]_{+}}^{m+n}\left(\begin{array}{c}
m+n \\
j
\end{array}\right)\left(\begin{array}{c}
m+n+j \\
2 m
\end{array}\right) \frac{E_{n-m+j}}{2^{n-m+j}}=\left(-\frac{1}{4}\right)^{n}\left(\begin{array}{c}
m+n \\
m
\end{array}\right) .
$$


The theorem follows by substituting $j=m-n+k$.

Again we have the following two interesting special cases.

COROLlary 2. For an integer $n \geqslant 1$

$$
\begin{gathered}
\sum_{k=n}^{2 n}\left(\begin{array}{c}
n \\
k-n
\end{array}\right) \frac{E_{k}}{2^{k}}=\left(-\frac{1}{4}\right)^{n} \\
\sum_{k=0}^{2 n}\left(\begin{array}{c}
2 n+k \\
2 k
\end{array}\right)\left(\begin{array}{c}
2 k \\
k
\end{array}\right) \frac{E_{k}}{2^{k}}=\left(-\frac{1}{4}\right)^{n}\left(\begin{array}{c}
2 n \\
n
\end{array}\right) .
\end{gathered}
$$

In deriving (b), we use the equality

$$
\left(\begin{array}{c}
2 n \\
k
\end{array}\right)\left(\begin{array}{c}
2 n+k \\
k
\end{array}\right)=\left(\begin{array}{c}
2 n+k \\
2 k
\end{array}\right)\left(\begin{array}{c}
2 k \\
k
\end{array}\right)
$$

We may compare the recurrence relations for Bernoulli and Euler numbers in (2) with those given in Corollaries 1 and 2 .

Finally we recall that the Genocchi numbers may be defined by $G_{0}=0$ and $G_{n}=n E_{n-1}(0)(n=1,2, \ldots)$. We refer to [4] for an interesting exposition on $G_{n}$ and related polynomials and to [2, p.49] for a table of the first few Genocchi numbers. It follows from the second formula in (1) that the Genocchi numbers satisfy the recurrence relation

$$
2 G_{n}+\sum_{k=0}^{n-1}\left(\begin{array}{l}
n \\
k
\end{array}\right) G_{k}=0 \quad(n \geqslant 2) .
$$

THEOREM 3. Let $n \geqslant 1$ be an integer. Then for any integer $m \geqslant 0$

$$
\begin{aligned}
\sum_{k=[n-m]_{+}}^{2 n}\left(\begin{array}{c}
m+n \\
m-n+k
\end{array}\right)\left(\begin{array}{c}
2 m+k \\
k
\end{array}\right) G_{k} & =0 \\
\sum_{k=[n-m]_{+}}^{2 n}\left(\begin{array}{c}
m+n+1 \\
m-n+k
\end{array}\right)\left(\begin{array}{c}
2 m+k+1 \\
k
\end{array}\right) \frac{G_{k}}{2^{k}} & =(-1)^{n} \frac{m+1}{2^{2 n}}\left(\begin{array}{c}
m+n+1 \\
n
\end{array}\right) .
\end{aligned}
$$

Proof: Let $m \geqslant 0$ be given. By Lemma 1 the $(2 m+1)^{\text {th }}$ derivative of $Q_{m+n}(x)$ vanishes at $x=0$. So (a) follows by a calculation similar to that in the proof of Theorem 2 and using the equality

$$
\left(\begin{array}{l}
2 m+k \\
2 m+1
\end{array}\right)=\frac{k}{2 m+1}\left(\begin{array}{c}
2 m+k \\
k
\end{array}\right)
$$

On the other hand, (b) follows directly from the two formulas obtained in Theorem 1 , as $G_{n}=2\left(1-2^{n}\right) B_{n}$ for $n \geqslant 0$.

In particular we have the following consequences. 
Corollary 3. For an integer $n \geqslant 1$

(a)

$$
\begin{gathered}
\sum_{k=n}^{2 n}\left(\begin{array}{c}
n \\
k-n
\end{array}\right) G_{k}=0 \\
\sum_{k=n}^{2 n}\left(\begin{array}{c}
n+1 \\
k-n
\end{array}\right)(k+1) \frac{G_{k}}{2^{k}}=(-1)^{n} \frac{n+1}{2^{2 n}} \\
\sum_{k=1}^{2 n}\left(\begin{array}{c}
2 n+k \\
2 k
\end{array}\right)\left(\begin{array}{c}
2 k \\
k
\end{array}\right) G_{k}=0 ; \\
\sum_{k=0}^{2 n}\left(\begin{array}{c}
2 n+k+1 \\
2 k
\end{array}\right)\left(\begin{array}{c}
2 k \\
k
\end{array}\right) \frac{G_{k}}{2^{k}}=(-1)^{n} \frac{n+1}{2^{2 n}}\left(\begin{array}{c}
2 n+1 \\
n
\end{array}\right)
\end{gathered}
$$

In deriving (c) and (d), we use again (10) and (11). We may compare (12) with those given in Corollary 3 .

\section{REFERENCES}

[1] M. Abramowitz and A. Stegun, Handbook of mathematical functions, Appl. Math. Ser. 55 (National Bureau of Standards, Washington D.C., 1972).

[2] L. Comtet, Advanced combinatorics (Reidel, Dordrecht and Boston, 1974).

[3] K. Dilcher, 'Sums and products of Bernoulli numbers', J. Number Theory 60 (1996), 23-41.

[4] A.F. Horadam, 'Genocchi polynomials', in Applications of Fibonacci numbers, (G.E. Bergum et al., Editor) (Kluwer Academic Publishers, Dordrecht, 1991), pp. 145-166.

[5] F.T. Howard, 'Applications of a recurrence for the Bernoulli numbers', J. Number Theory 52 (1995), 157-172.

[6] M. Kaneko, 'A recurrence formula for the Bernoulli numbers', Proc. Japan Acad. Ser. A 71 (1995), 192-193.

[7] J. Riordan, Combinatorial identities (R.E. Krieger Publishing Co., New York, 1979).

[8] J. Satoh, 'A recurrence formula for $q$-Bernoulli numbers attached to formal group', Nagoya Math. J. 157 (2000), 93-101.

Department of Mathematics

Hualien Teachers College

Hualien

Taiwa

e-mail: chchang@sparc2.nhltc.edu.tw
Department of Mathematics

Tsing Hua University

Hsinchu

Taiwan

e-mail: cwha@math.nthu.edu.tw 